\title{
Pregnancy-Associated Malaria in Sudan: Prevalence and Possible Risk Factors
}

\author{
Samia A. Omer ${ }^{*}, 1$, Eltahir Awad Gasim Khalil ${ }^{2}$, Abdalla Hassan Sharief ${ }^{1}$ and \\ Hashim Abdraham Ali ${ }^{3}$ \\ ${ }^{I}$ Tropical Medicine Research Institute, National Centre for Research, Khartoum, Sudan \\ ${ }^{2}$ Institute of Endemic Diseases, University of Khartoum, Sudan \\ ${ }^{3}$ Omdurman Maternity Hospital, Omdurman, Sudan
}

\begin{abstract}
This study aimed to investigate the malaria infection prevalence and predisposing risk factors among some pregnant women in Greater Khartoum area, Sudan. Following informed consent, eight hundred thirty six pregnant women were interviewed and thick blood films were prepared and examined for malaria parasites. Parasite densities were determined by counting parasites against 200 leukocytes assuming a white blood cells count of $6,000 / \mu 1$. PCR was performed using outer and nested primers to enhance detection of parasitemia below the microscopy threshold, after DNA extraction by chelex method. Microscopy and species-specific PCR results showed that $26.2 \%$ and $56.5 \%$ of women were positive respectively. Low parity and young-aged pregnant women were significantly associated with parasitemia $(p=0.001,0.004)$. Second trimester pregnancy [adjusted odd ratio (aOR), 3.2 (95\% CI, 2.9-5.8,)] and season of antenatal visit [(aOR), 1.7 (95\% CI, (0.9-1.1)] constituted a significant risk factor for contracting malaria. In a multivariate analysis, mothers $<20$ years [(aOR), 2.4 (95\% CI, 1.3-3.0)], first and second pregnancies [(aOR), 2.0 (95\% CI, 1.1-1.9)], povertyrelated factors (low-income, illiteracy) were independent predictors for $P$. falciparum infection. Pregnancy-associated $P$. falciparum malaria is prevalent among Sudanese pregnant women with evidence of close correlation to low parity, young age and socioeconomic status.
\end{abstract}

Keywords: Malaria, risk factors, pregnant women, Sudan.

\section{INTRODUCTION}

It has long been observed that there is an increase in the severity of malaria during pregnancy, resulting in certain negative outcomes as maternal anemia and low birth weight, with an increase in maternal and infant mortality [1,2]. Pregnancy-associated malaria is a cause of morbidity and mortality to mothers and their developing fetuses in subSaharan Africa, where it is well documented that pregnant women are more susceptible to malaria infection compared to before pregnancy and to their non-pregnant counterparts. It is known that the key malaria interventions are vector control using insecticide-treated nets (ITNs), indoor residual spraying (IRS), intermittent preventive treatment of malaria in pregnancy (IPTp) and effective treatment. Artemisininbased combination therapy (ACT). Between 2000 and 2006, ITN distribution increased three- to ten-fold in most countries including Sudan. Subsidized or free ITNs have increased bednet coverage. ITN distribution is linked to antenatal care.

In addition, P. falciparum malaria is a particular danger in pregnancy and can have significant adverse consequences for both the mother and the developing fetus, including

*Address correspondence to this author at the Tropical Medicine Research Institute, National Centre for Research, Khartoum, Sudan; Tel: + 249128 74286; Fax: + 249183781845

E-mail: samiaomer@hotmail.com abortion, stillbirth, low birth weight and maternal anemia [25]. The increased susceptibility of pregnant women to $P$. falciparum infection is partially due to physiological alterations of immunity. Moreover, clinical malaria has been attributed to specific strains of $P$. falciparum that are able to adhere to chondroitin sulphate $\mathrm{A}$ in the placenta [6].

In endemic areas, the prevalence of clinical and asymptomatic malaria is highest in young women and those in their first and second pregnancies. The vulnerability decreases, however, with increasing numbers of pregnancies suggesting that women acquire a gravidity-form of immunity, resulting in a decrease in both prevalence and severity of the disease $[7,8]$. The prevalence of malaria infection and parasite densities in pregnant women are reported to be highest throughout the second trimester [4, 9]. Regardless of the pre-pregnancy level of immunity against malaria, the most frequent consequence of malaria during pregnancy is anemia. Several studies have reported malaria infection as the primary cause for anemia among pregnant women, and the changes in the immune system associated with pregnancy increasing the susceptibility to anemia $[7,10,11]$. Many studies pointed to alarming the prevalence of malaria in pregnant women in several African countries, only a hand full of studies have evaluated such situation in Sudan [5, 12].

In this study, we aimed to investigate the malaria prevalence and its predisposing risk factors during pregnancy 
among Sudanese pregnant women in Greater Khartoum area, Sudan.

\section{MATERIALS AND METHODS}

\section{Study Site}

During the period from July 2003 to December 2004, pregnant women attending the routine antenatal clinics at Omdurman Maternity Hospital were enrolled. Omdurman Maternity Hospital is a major public hospital serves residents of around 5 million people in central Sudan. Omdurman city has a mixed rural, tribal, and urban population. The Study protocol was reviewed by the ethical and scientific committees of the Institute of Endemic Diseases, University of Khartoum and the Directorate of Research, Federal Ministry of Health, Khartoum, Sudan.

\section{Data Collection}

Enrolled volunteers were consented and a pre-designed questionnaire that included socio-demographic data, information on maternal age, gravidity and gestation was completed. Volunteer ethnicity, residence and income to past history of malaria and the use of anti malarial drugs during pregnancy were also included. Women were scheduled for follow up visits on a monthly basis until delivery. Gravidity was categorized as primigravidae, secundigravidae, multigraviade (gravida 3-5) and grand multigraviad (gravida $\geq 6$ ). The following age groups were used: teenagers $(<20$ years old), young women (20-24 years old) and older women ( $>24$ years old). Length of gestation was estimated based on the number of weeks between the self-reported date of last menstrual period (LMP) and antenatal visit date. Trimesters were defined as first ( $<14$ weeks), second (14-27 weeks) and third ( $>27$ weeks) and residence was classified as urban, semi-urban and rural areas; women at the 3 sites were compared. Women attending the antenatal clinic were given one month's supply of iron (II) sulfate tablets and folic acid tablets. Similarly, expected date of delivery for each participant was also calculated.

\section{Laboratory Tests}

Collection of samples was based on the seasonality of malaria prevalence in the various seasons of the year. The rainy season usually starts in July and continued to October. Particularly the weather in Sudan characterized by a 3-month following the rainfall season known as post rainy season and this extends from November to early February followed by the dry season in the remaining (March-June) period.

Blood samples were collected, at each visit, for parasitological and molecular evaluations. Finger prick blood samples were collected for thick and thin blood films and DNA extraction on No. 3 whatman filter paper. Films were stained with 3\% Giemsa and 100-oil immersion fields were examined microscopically by two independent technicians. Parasite densities were determined by counting parasites and 200 leucocytes assuming white blood cells count of $6,000 / \mu 1$. Parasitemia were graded as low $(1-999 \mu 1)$, moderate $(1000-$ $9999 / \mu \mathrm{l})$ and high $(>10000 / \mu \mathrm{l})$. Hemoglobin $(\mathrm{Hb})$ was mea- sured using a colorimetric method and $\mathrm{Hb}$ levels were defined as normal and anemic $(<11 \mathrm{~g} / \mathrm{dl})$.

\section{Parasite DNA Extraction and PCR Amplification}

DNA was extracted from filter papers using chelex method as described by Plowe et al. [13]. PCR was carried out using outer and nested primers to enhance detection of parasitemia below the microscopy threshold. All samples were screened for $P$. falciparum DNA using nested PCR amplification of the small subunit ribosomal RNA gene, as previously described [14]. The amplified DNA products were then analyzed electrophoretically on $1.5 \%$ agrose gels and stained with ethidium bromide. Bands were visualized under UV trans-illumination, and photographed.

\section{Statistical Analysis}

Following data entry, rechecking and verification, data were analyzed using Epi-Info 2002 software. Chi-square analysis and Fisher exact test were used to compare proportions within and among groups. Logistic regressions were performed with malaria as the dependent variable, using age, gravidity, season and gestation as independent variables. Ninety five percent (95\%) confidence intervals (CIs) were considered.

\section{RESULTS}

\section{Description of Study Population}

The majority of pregnant women were between 21-30 years of age with women $\leq 20$ years constituting less than

Table 1. Baseline Characteristics of the Study Population $(\mathbf{n}=\mathbf{8 3 6})$

\begin{tabular}{|c|c|}
\hline Characteristics & Number (\%) \\
\hline \multicolumn{2}{|l|}{ Age groups: } \\
\hline$\leq 20$ & $154(18.5 \%)$ \\
\hline $21-25$ & $263(31.3 \%)$ \\
\hline $26-30$ & $238(28.5 \%)$ \\
\hline$>30$ & $181(21.7 \%)$ \\
\hline \multicolumn{2}{|l|}{ Gravidity: } \\
\hline Primigravidae & $355(42.5 \%)$ \\
\hline Secundigravidae & $238(28.5 \%)$ \\
\hline multigraviadae & $139(16.6 \%)$ \\
\hline Grand multigraveida & $104(12.4 \%)$ \\
\hline \multicolumn{2}{|l|}{ Gestational age at recruitment: } \\
\hline First trimester & $306(36.6 \%)$ \\
\hline Second trimester & $402(48.1 \%)$ \\
\hline Third trimester & $128(15.3 \%)$ \\
\hline \multicolumn{2}{|l|}{ Residence: } \\
\hline Urban & $142(17.1 \%)$ \\
\hline Semi urban & $426(51.3 \%)$ \\
\hline Rural & $268(31.6 \%)$ \\
\hline \multicolumn{2}{|l|}{ Educational level: } \\
\hline Able to read & $542(64.8 \%)$ \\
\hline Illiterate & $294(35.2 \%)$ \\
\hline Anemic $(\mathrm{Hg}<11)$ & $557(66.6 \%)$ \\
\hline
\end{tabular}


Table 2. Prevalence of Pregnancy-Associated Malaria by Maternal Age and Gravidity ( $\mathrm{n}=836$ )

\begin{tabular}{|c|c|c|c|c|c|c|}
\hline & \multicolumn{3}{|c|}{ Maternal Age (years) } & \multicolumn{3}{|c|}{ No. of Pregnancies } \\
\hline & $\leq 20$ & $>20$ & $p$ & $1-2$ & $\geq 3$ & $p$ \\
\hline $\begin{array}{c}\frac{\text { Malaria }}{\text { positive }} \\
\text { Microscopy } \\
(\mathrm{n}=219)\end{array}$ & $\begin{array}{c}59.7 \% \\
(92 / 154)\end{array}$ & $\begin{array}{c}18.6 \% \\
(127 / 682)\end{array}$ & 0.004 & $\begin{array}{c}30.2 \% \\
(179 / 593)\end{array}$ & $\begin{array}{c}16.1 \% \\
(40 / 243)\end{array}$ & 0.001 \\
\hline $\begin{array}{c}\text { PCR } \\
(\mathrm{n}=471)\end{array}$ & $\begin{array}{c}70 \% \\
(107 / 154)\end{array}$ & $\begin{array}{c}57.8 \% \\
(394 / 684)\end{array}$ & 0.02 & $\begin{array}{c}40.8 \% \\
(242 / 593)\end{array}$ & $\begin{array}{c}62.6 \% \\
(152 / 243)\end{array}$ & 0.025 \\
\hline
\end{tabular}

$20 \%$ of the study volunteers. About half of women attended in the second trimester $(48.1 \%)$ with few attendees in the third trimester $(15.3 \%)$. Almost half $(51.3 \%)$ of the women came from semi-urban areas $(p=0.06)$. The proportion of primigravidae was higher among those who came from urban areas. Illiteracy was detected in nearly third (30.1\%) of the study group. Most of the pregnant women (66.6\%) had hemoglobin levels less than 11.0 grams/dL (i.e. anemic) (Table 1).

\section{Malaria Prevalence by Age and Gravidity}

Microscopy and species-specific primers PCR diagnosis showed that $26.2 \%(219 / 836)$ and $56.3 \%(471 / 836)$ of women were positive respectively, with $P$. falciparum as the dominant spp. Both microscopy and PCR showed significant malaria prevalence among women $\leq 20$ years of age compared to those over 20 years $(p<0.005)$. However, gravidity was associated with a decrease in the prevalence of malaria detected by microscopy (Table 2 ). There was malarial infec- tion in $30.2 \%$ of pucigravidae (women $\leq 2$ pregnancies) compared to $16.1 \%$ in multigravidae $(p=0.001)$. The malaria prevalence declined as women became older. Infection among primigravidae was associated with higher parasitaemia compared to that among multigraviade $(p=0.003)$.

\section{Predisposing Factors for Malaria Infection}

Malarial infection was significantly higher in women from rural and semi-urban areas compared to woman from urban areas $(p<0.003)$. The microscopic frequencies of malaria infection among women from various areas were $35.1 \%, 27.2 \%$ and $6.3 \%$ for rural, semi-urban and urban areas respectively, while the PCR frequencies were $78.6 \%$, $56.8 \%$ and $14.1 \%$ for the same areas respectively.

Univariate analysis showed malaria risk factors to include: maternal age " $p=0.001 "$, gravidity " $p=0.004 "$ and trimester and living in rural areas " $p<0.0001 "$ ) as diagnosed by blood films. After correlating other variables in a multiva-

Table 3. Risk Factors for Malaria Infection (Positive Blood Film)

\begin{tabular}{|c|c|c|c|c|c|}
\hline \multirow{2}{*}{ Risk Variable } & \multirow{2}{*}{ No. Infected (\%) } & \multicolumn{2}{|c|}{ Unadjusted OR } & \multicolumn{2}{|c|}{ Adjusted OR } \\
\hline & & $(95 \% \mathrm{CI})$ & $p$ & $(95 \% \mathrm{CI})$ & $p$ \\
\hline Age (years): & & & & & \\
\hline$\leq 20$ & $92 / 154(95.7 \%)$ & $2.9(2.4-3.6)$ & 0.0002 & $2.4(1.3-3.0)$ & 0.06 \\
\hline $21-25$ & $61 / 263(23.3 \%)$ & $1.5(1.3-2.1)$ & 0.008 & $2.0(1.3-1.8)$ & 0.002 \\
\hline $25-29$ & $46 / 238(19.2 \%)$ & 1 & & 1 & \\
\hline$>30$ & $20 / 181(11.0 \%)$ & $0.6(0.9-1.1)$ & 0.03 & $1.1(0.9-1.4)$ & 0.001 \\
\hline Gravidaty: & & & & & \\
\hline Pucigravidae & $179 / 593(30.2 \%)$ & $2.5(1.5-2.8)$ & 0.0001 & $2.0(1.1-1.9)$ & 0.001 \\
\hline Multigravidae & $28 / 139(20.7 \%)$ & 1 & & 1 & \\
\hline Grandmultigravidae & $12 / 104(11.0 \%)$ & $0.8(0.7-1.1)$ & 0.02 & $0.1(0.2-0.5)$ & 0.03 \\
\hline Trimester: & & & & & \\
\hline First & $34 / 306(11.1 \%) 1$ & 1 & & 1 & \\
\hline Second & $162 / 402(48.3 \%)$ & $2.9(2.0-6)$ & 0.006 & $3.2(2.9-5.8)$ & 0.0008 \\
\hline Third & $23 / 128(17.8 \%)$ & $2.1(0.5-1.6)$ & 0.03 & $0.9(1.2-2.6)$ & 0.04 \\
\hline Season of antenatal visit: & & & & & \\
\hline Rainy season & $180 / 523(34.4 \%)$ & $1.3(0.8-1.0)$ & 0.05 & $1.7(0.9-1.1)$ & 0.01 \\
\hline Dry season & 29/313 (9.3\%) & 1 & & 1 & \\
\hline Socio-economic class: & & & & & \\
\hline Low & $173 / 462(37.4 \%)$ & $1.7(1.0-2.1)$ & 0.002 & $2.3(1.3-4.0)$ & 0.001 \\
\hline High & $46 / 374(12.3 \%)$ & 1 & & 1 & \\
\hline
\end{tabular}




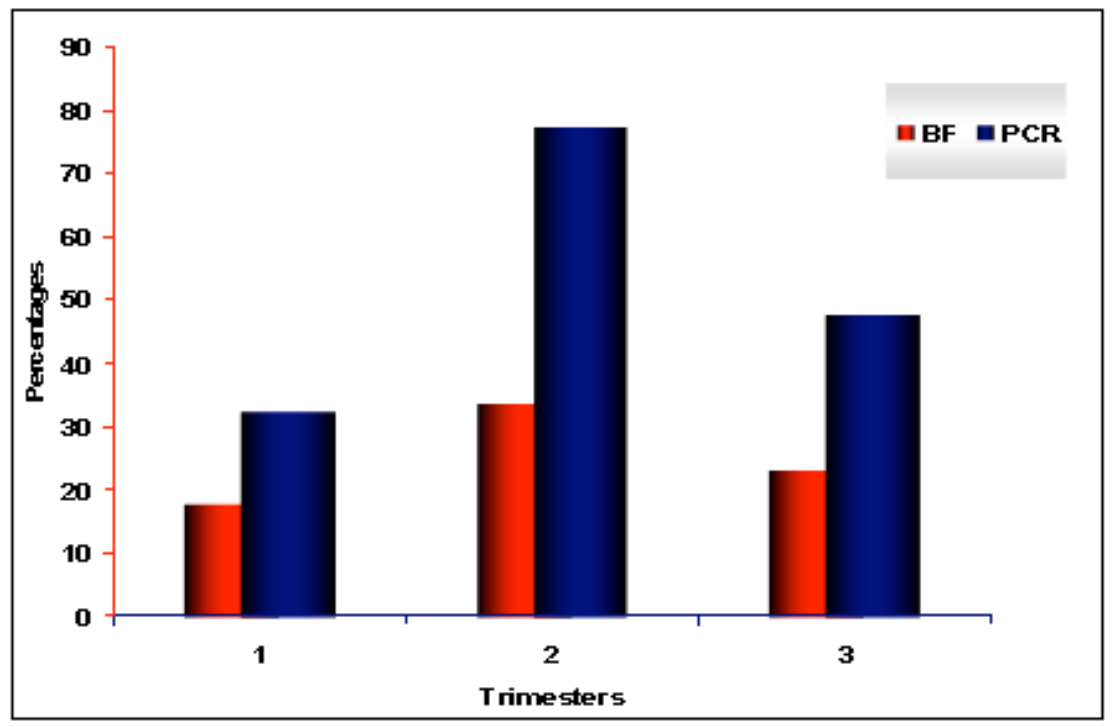

Fig. (1). Prevalence of pregnancy associated malaria by microscopy and PCR in all trimester.

riate analysis and by microscopic detection, pucigravidae, below 20 years, trimester and season were independently associated with malaria infection (Table 3 ).

Infection rates were higher among those in the second trimester, by both microscopy and PCR (33.1\% and $77.4 \%)$, followed by women in the third trimester and then those in the first one (Fig. 1). Women with microscopic positivity were more symptomatic compared to those who were diagnosed by PCR and those who were negative for malaria parasite $(90.4 \%, 33.8 \%$ and $13.9 \%$ respectively). Ethnicity was not associated with increased malaria infection; an approximate equal proportion was noticed $(49.1 \%$ in non-Arab and $50.9 \%$ for Arabs $(p=0.7)$.

Malaria positivity (both by microscopy and PCR) in antenatal visits varied by season and was highest during and after the rainy season with averages of $15.3 \%$ and $29.6 \%$ compared to $2.3 \%$ and $6.4 \%$ in the post rainy dry season $(p=$
$0.002, p=0.001)$ (Fig. 2). There was a strong correlation between the occurrence of anemia and positive blood films, where $34.4 \%$ of anemic women were parasitemic compared to $7.3 \%$ of women with normal hemoglobin level $(p=0.0001)$. The mean hemoglobin levels decreased significantly as parasite counts increased $(p<0.01)$. The prevalence of anemia was higher among pregnant women in their second and third trimesters compared to those in their first trimester $(p=0.02)$. Anemia prevalence was higher in women living in rural and semi-urban areas compared to those from urban areas 63.1\% (168/286), $44.6 \%(190 / 426)$ and $20 \%$ $(29 / 142)$ respectively $(p<0.05)$.

\section{DISCUSSION}

It is well documented that pregnant women living in many parts of sub-Saharan Africa have an increased risk of

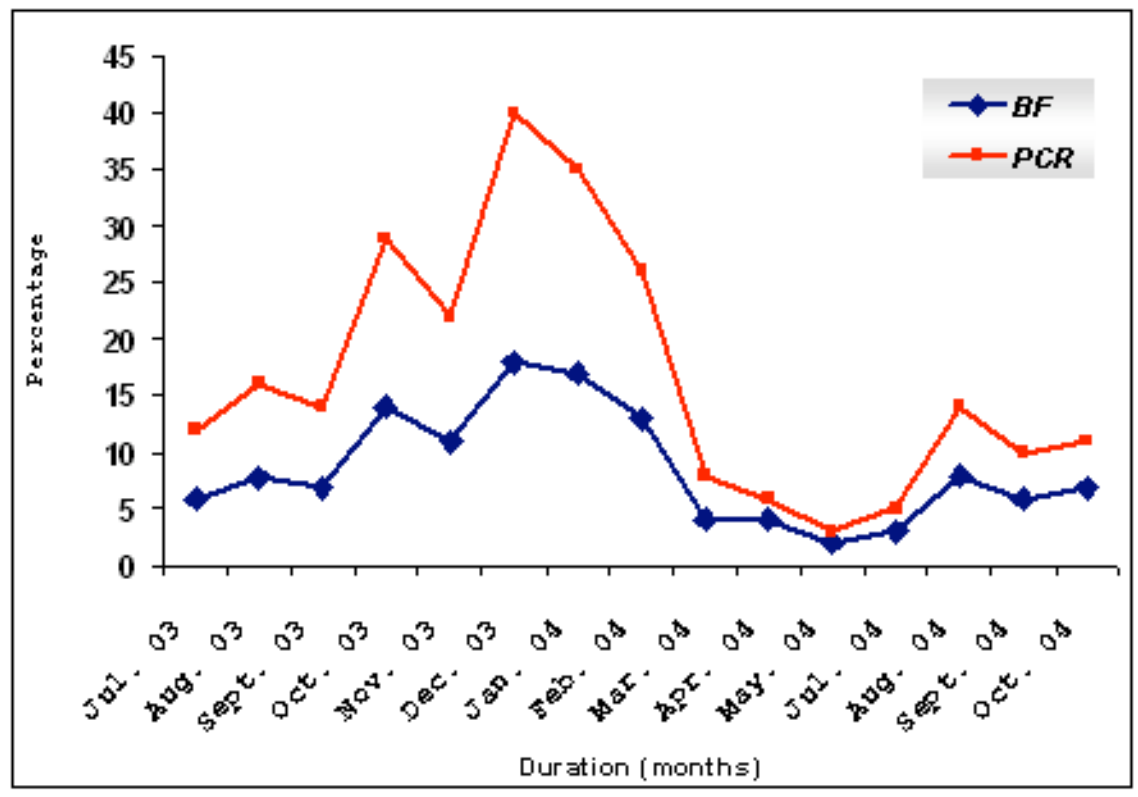

Fig. (2). Prevalence of pregnancy associated malaria by microscopy and PCR during the study period (July 03-Oct. 04). 
malaria infection, but limited data have been reported from Sudan $[5,12]$. Sudan is a vast country with more than two hundreds ethnic groups which resulted mainly from AfroArab admixture. This study showed that malaria prevalence varies in different trimesters, a finding that has been reported in other parts of the world with varied malaria endemicity [3, $4,7,8,19]$. The risk of infection was higher among pucigravidae particularly among women below 25 years of age. This could be explained by the fact that acquisition of immunity to malaria increases with successive pregnancies. Such immunity is thought to be due either to priming of memory $\mathrm{T}$ cells or to production of antibodies against $P$. falciparum chondroitin sulfate A (PfCSA) that prevent parasites' sequestration in the placenta $[15,16,21]$. The study also indicated that malaria parasite density decreased with increasing parity and age further reinforcing the belief that the ability to control malaria parasitemia is parity and/or agedependent.

Parasitemia was more frequent during the second trimester, probably points to the time of maximum malaria risk during pregnancy $[4,9,17]$. The present study indicated that the malaria risks could also be poverty-related like illiteracy; low income and residence in rural areas, where health care facilities and malaria control programmes are meager and less effective. Women living in urban areas have access to adequate health care and malaria control facilities as well as their better health education $[9,18]$.

Our study confirmed the facts that, the overall prevalence of microscopic parasitaemia had seasonal variation, being highest at the post rainy season $[4,20]$.

In conclusion, pregnancy-associated $P$. falciparum malaria is prevalent among Sudanese pregnant women with seasonal variations. There is close correlation between malaria prevalence of malaria among pregnant women and the socioeconomic status.

\section{ACKNOWLEDGEMENT}

The contribution of the volunteers and the staff of Omdurman Maternity Hospital is gratefully acknowledged. We Thank Dr. Hamza A. Babiker for his help and support. PCR assays were done at the Institute of Immunology and Infection Research, Edinburgh University, Edinburgh. This work received financial support from The Gordon Memorial Trust Fund, UK and the National Centre for Research, Sudan.

\section{REFERENCES}

[1] McGregor IA. Epidemiology, malaria and pregnancy. Am J Trop Med Hyg 1984; 33: 517-25.
[2] Brabin B. An analysis of malaria in pregnancy in Africa. Bull WHO 1983; 61: 1005-16.

[3] Mendez F, Carrasquilla G, Munoz A. Risk factors associated with malaria infection in an urban setting. Trans R Soc Trop Med Hyg 2000; 94(4): 367-71.

[4] Dicko A, Mantel C, Aly Thera M, et al. Risk factors for malaria infection and anemia for pregnant women in the Sahel area of Bandiagara, Mali. Acta Trop 2003; 89(1): 17-23.

[5] Adam I, Khamis A, Elbashir M. Prevalence and risk factors for Plasmodium falciparum malaria in pregnant women of eastern Sudan. Malar J 2005; 4: 18.

[6] Fried M, Duffy P. Adherence of Plasmodium falciparum to Chondroitin Sulfate A in the human placenta. Science 1996; 272: 1502-4.

[7] Bouyou-Akotet M, Ionete-Collard D, Mabika-Manfoumbi M, et al. Prevalence of Plasmodium falciparum infection in pregnant women in Gabon. Malar J 2003; 2: 18.

[8] Walker-Abbey A, Djokam R, Eno A, et al. Malaria in pregnant Cameroonian women: The effect of Age and gravidity on submicroscopic and mixed-species infections and multiple parasite genotypes. Am J Trop Med Hyg 2005; 72(3): 229-35.

[9] Zhou A, Megnekou R, Leke R, et al. Prevalence of Plasmodium falciparum infection in pregnant Cameroonian women. Am J Trop Med Hyg 2002; 67(6): 566-70.

[10] Menedenz C. Malaria during pregnancy: a priority area of malaria research and control. Parasitol Today 1995; 11(5): 178-83.

[11] Glover-Amengor M, Owusu W, Akanmori B. Determinants of anemia in pregnancy in Sekyere west district, Ghana. Ghana Med J 2005; 39(3): 102-7.

[12] Adam I, Khamis A, Elbashir M. Prevalence and risk factors for anemia in pregnant women of eastern Sudan. Trans R Soc Trop Med Hyg 2005; 99(10): 739-43.

[13] Plowe C, Djimde A, Bouare M, Doumbo O, Wellems T. Pyrimethamine and proguanil resistance conferring mutations in Plasmodium falciparum dihydrofolate reductase; polymerase chain reaction methods for surveillance in Africa. Am J Trop Med Hyg 1995; 52(6): 565-8.

[14] Snounou G, Viriyakosol S, Zhu X, et al. High sensitivity of detection of human malaria parasites by the use of nested polymerase chain reaction. Mol Biochem Parasitol 1993; 61(2): 315-20.

[15] Maubert B, Fievet N, Tami G, Cot M, Boudin C, Deloron P. Development of antibodies against Chondroitin Sulfate A-adherent Plasmodium falciparum in pregnant women. Infect Immun 1999; 67(10): 5367-71.

[16] Moore J, Nahlen B, Lala A. Udhayakumar V. Immunologic memory in the placenta: a lymphocyte recirculation hypothesis. Med Hypotheses 2000; 54(3): 505-10.

[17] Nosten F, ter kuile F, Maelankirri L, Decludt B, White N. Malaria during pregnancy in an area of unstable endemicity. Trans R Soc Trop Med Hyg 1991; 85(4): 424-9.

[18] Silver HM. Malarial infection during pregnancy. Infect Obstet 1997; 11: 99-107.

[19] Granja C, Machungo F, Gomes A, Bergstrom S, Brabin B. Malariarelated maternal mortality in urban Mozambique. Ann Trop Med Parasitol 1998; 92: 257-63.

[20] Saute F, Menendez C, Mayor A, et al. Malaria in pregnancy in rural Mozambique: the role of parity, submicroscopic and multiple plasmodium falciparum infections. Trop Med Int Health 2002; 7(1): 19-28.

[21] Fleming AF. The aetiology of severe anemia in pregnancy in Ndole, Zambia. Ann Trop Med Parasitol 1989; 83: 37-49. 